\title{
A UNIVERSAL ARONSZAJN LINE
}

\author{
Justin Tatch Moore
}

\begin{abstract}
The purpose of this note is to define an Aronszajn line $\eta_{C}$ and prove that under the assumption of PFA it is universal for the class of Aronszajn lines. Moreover $\eta_{C}$ can be easily described in terms of a fixed Countryman type $C$ : it is the direct limit of the finite lexicographic products of the form $C \times(-C) \times \ldots \times( \pm C)$.
\end{abstract}

\section{Introduction}

An uncountable linear order is Aronszajn if it has no uncountable separable suborders and does not contain a copy of $\omega_{1}$ or $-\omega_{1}$. These linear orders were considered and proved to exist by Aronszajn and Kurepa [8] (see also $[15, \S 5]$ ) in the course of studying Souslin's Problem [14]. One of the main difficulties in dealing with this class lies in the fact that it is defined in terms of what order types its members do not contain. Hence its members are typically dealt with in a fairly abstract manner.

In this paper I will use the Proper Forcing Axiom (PFA) to prove that all Aronszajn lines are isomorphic to a suborder of a single canonical Aronszajn line $\eta_{C}$. Here PFA is a strong Baire category assumption due to Baumgartner. It extends $\mathrm{MA}_{\aleph_{1}}$ to a broader class of partial orders and is a natural assumption in this context (see [1], [3], $[17, \S 8],[19],[20])$. The universality of $\eta_{C}$ and the results of this paper also hold in Woodin's canonical model for the failure of the Continuum Hypothesis [22].

In [10] it was proved that, assuming PFA, the Aronszajn lines contain a two element basis. That is, there are two Aronszajn lines such that any other contains an isomorphic copy of one of these two. The elements of this basis have the following property: their Cartesian square can be covered by countably many non decreasing relations. Such orders are said to be Countryman and were first constructed by Shelah in [12]. Unlike being Aronszajn, being Countryman is phrased in terms of the existence of a simple global structure on the linear order. Remarkably, the existence of such a structure automatically entails that the order is Aronszajn. A further important observation is that if $C$ is Countryman and $-C$ is its reverse, then no uncountable linear order can embed into both $C$ and $-C$. In particular, the Aronszajn lines cannot have a single element basis.

In [16], Todorcevic produced a number of concrete representations of Countryman lines (see also [21]). He moreover proved that such linear orders are canonical in the

Received by the editors February 26, 2008.

2000 Mathematics Subject Classification. Primary: 03E75, 06A05; Secondary: 03E05, 03E35, $03 \mathrm{E} 65$.

Key words and phrases. Aronszajn, Countryman, linear order, PFA, universal. 
presence of $\mathrm{MA}_{\aleph_{1}}$. The following is a strong form of $[21,2.1 .12]$ which is proved by a similar argument. ${ }^{1}$

Theorem 1.1. $\left(\mathrm{MA}_{\aleph_{1}}\right)$ Every two $\aleph_{1}$-dense non stationary Countryman lines are either isomorphic or reverse isomorphic. In particular if $C$ is a Countryman line, then $C$ and $-C$ form a two element basis for all Countryman lines.

The construction of $\eta_{C}$ is as follows. Fix a Countyman line $C$ and let $\zeta_{C}$ denote the direct sum $-C \oplus\{0\} \oplus C$. The order $\eta_{C}$ is the subset of the lexicographic power $\left(\zeta_{C}\right)^{\omega}$ consisting of those elements which are eventually 0 .

Theorem 1.2. (PFA) Every Aronszajn line is isomorphic to a suborder of $\eta_{C}$.

Notice that if we let $\eta$ denote the ordertype of $\mathbb{Q}$, then we have a rather strong analogy between the relationship of $\mathbb{N}$ and $-\mathbb{N}$ to $\eta$ and the relationship of $C$ and $-C$ to $\eta_{C}$.

In the course of proving the main result, I will also establish the following result which is of independent interest.

Theorem 1.3. (PFA) If $A$ is an Aronszajn line which is not Countryman, then $A$ contains an isomorphic copy of both $C$ and $-C$ for some (equivalently any) Countryman line $C$.

As this result is proved, it will be remarked how the argument can be adapted to yield a complete proof of Theorem 1.1.

This paper is intended to be fairly self contained, although I will cite some established consequences of PFA at appropriate points. The reader is, however, assumed to have some familiarity with set theory - [7] is a standard reference (chapter 2 is particularly relevant). The reader should recall in particular that ordinals are taken, in the sense of von Neumann, to be the set of their predecessors. A sequence is a function whose domain is an ordinal. Further information on the Proper Forcing Axiom and associated arguments can be found in [4], [13], and [17]. The reader can find additional information on trees and linear orders in [15].

\section{Preliminary analysis}

It will be helpful to first define some notation and make some observations about Aronszajn lines. First, recall that every Aronszajn line has cardinality $\aleph_{1}[15, \S 5]$ and hence can be represented as a linear ordering on $\omega_{1}$. Let $A$ be a fixed Aronszajn ordering on $\omega_{1}$. A can be represented as a lexicographic ordering on a subset of $2^{\omega_{1}}$ in the following way. For each $\alpha<\omega_{1}$, let $a_{\alpha}: \omega_{1} \rightarrow 2$ be the characteristic function of the set of $\xi<\omega_{1}$ such that $\xi<_{A} \alpha$. It is easy to see that the function sending $\alpha$ to $a_{\alpha}$ preserves order if its domain is given the order from $A$ and its range is given the lexicographic order.

If $\alpha \neq \beta$, it will be useful to let $\Delta_{A}(\alpha, \beta)$ denote the least $\xi$ such that $a_{\alpha}(\xi) \neq a_{\beta}(\xi)$. The following fact is routine to verify.

Fact 2.1. Suppose that $\alpha \leq_{A} \alpha^{\prime} \leq_{A} \beta^{\prime} \leq_{A} \beta$. Then $\Delta_{A}(\alpha, \beta) \leq \Delta_{A}\left(\alpha^{\prime}, \beta^{\prime}\right)$.

\footnotetext{
${ }^{1}$ I made an incorrect reference to this result in [11] by omitting "non stationary" from the statement. Without "non stationary" this theorem becomes false in a very strong sense [2] (see [15, 5.7]). See [18] for further information on the role of stationary sets in the context of Aronszajn lines.
} 
Notice that, since $A$ is Aronszajn, its elements have both countable cofinality and co-initiality. Consequently, for each $\alpha<\omega_{1}$, there is a least $\xi<\omega_{1}$ such that $\Delta_{A}(\alpha, \beta)<\xi$ whenever $\beta<\omega_{1}$. By letting $E$ be the set of fixed points of the operation $\alpha \mapsto \xi$, we have the following fact.

Fact 2.2. There is a club $E \subseteq \omega_{1}$ such that if $\alpha<\beta<\omega_{1}$, then $\Delta_{A}(\alpha, \beta)<$ $\min (E \backslash \alpha)$.

Let $T_{A}$ denote the set of all $a_{\xi}\left\lceil\eta\right.$ such that $\xi<\omega_{1}$ and $\eta \leq \min (E \backslash(\xi+1))$. In addition to its tree structure given by sequential extension, $T_{A}$ is also equipped with the lexicographic ordering which extends the ordering of sequential extension (i.e. if $s$ is an initial part of $t$, then $\left.s \leq_{\text {lex }} t\right)$. Notice that the $\operatorname{map} \xi \mapsto a_{\xi}\lceil\min (E \backslash(\xi+1))$ is an embedding of $A$ into $\left(T_{A}, \leq_{\text {lex }}\right)$.

Recall that a linear order $L$ is $\aleph_{1}$-dense if whenever $a<b$ are in $L \cup\{-\infty,+\infty\}$, the set of all $x$ in $L$ with $a<x<b$ has cardinality $\aleph_{1}$. Also, an Aronszajn line $A$ is non stationary if there is a continuous $\subseteq$-increasing sequence $\left\langle D_{\xi}: \xi<\omega_{1}\right\rangle$ of countable subsets of $A$ which cover $A$ and have the property that no maximal interval of $A \backslash D_{\xi}$ has a greatest or least element for any $\xi<\omega_{1}$. Stationarity of Aronszajn lines was introduced in [2] in order to generate a large number of pairwise non isomorphic Aronszajn lines in ZFC (see also [18]). The following is a standard fact about Aronszajn lines.

Fact 2.3. Every Aronszajn line contains an $\aleph_{1}$-dense suborder which is non stationary.

Finally, we will need the following fact about Countryman lines which follows easily from their definition.

Fact 2.4. If $C$ is Countryman, then $C$ does not contain a Souslin suborder. Equivalently, if $X$ is an uncountable subset of $T_{C}$, then $X$ contains an uncountable antichain.

\section{When Aronszajn lines are Countryman}

The purpose of this section is to prove Theorem 1.3 - that every non Countryman Aronszajn line must contain both "orientations" of a given Countryman line. At appropriate points in the argument, I will note how the proof can be adapted to yield a proof of Theorem 1.1. The proof in this section is similar to that of $[1, \S 5]$ and, e.g., $[21,2.1 .12]$. For completeness, I will present an argument which draws out the reasons why PFA is (likely) needed in the proof of Theorem 1.3 while $\mathrm{MA}_{\aleph_{1}}$ is sufficient for the proof of Theorem 1.1. I will include details which until now have been left to the reader.

For this section, let $A$ be a given Aronszajn line and $C$ be a given Countryman line. We will generally be interested in pairs $A$ and $C$ which satisfying the following hypothesis, but we will make explicit mention of when this property is needed:

H: Whenever $X$ is an uncountable suborder of $A, X$ contains a copy of $C$.

In particular, $(\mathrm{H})$ implies that $A$ does not contain a Souslin suborder. Without loss of generality, both $A$ and $C$ are orderings on the underlying set $\omega_{1}$. I will freely use the notation and observations collected in Section 2. 
Definition 3.1. Define $Q_{0}$ to be the collection of all finite partial functions from $A$ to $C$ which preserve order. This is considered as a forcing notion with the order of reverse inclusion. ${ }^{2}$

The forcing $Q_{0}$ fails to satisfy the countable chain condition for any number of reasons (which should become apparent momentarily). The following notion of [1] is what is needed to refine $Q_{0}$ to a collection which does satisfy the c.c.c..

Definition 3.2. If $f$ is a partial function from $A$ to $C$ and $\delta<\omega_{1}$, then $f$ is said to respect $\delta$ if:

(1) for all $\alpha$ in the domain of $f$,

$$
\alpha<\delta \Leftrightarrow f(\alpha)<\delta .
$$

(2) for all $\alpha \neq \beta$ in the domain of $f$,

$$
\Delta_{A}(\alpha, \beta)<\delta \Leftrightarrow \Delta_{C}(f(\alpha), f(\beta))<\delta .
$$

If $E$ is a subset of $\omega_{1}$, then $f$ respects $E$ if $f$ respects every element of $E$.

The following lemma gives an indication as to why this definition will play a role. It will be used in the verification of Theorem 1.1.

Lemma 3.3. If $R \subseteq A^{2}$ is a non decreasing relation, then there is a club $E \subseteq \omega_{1}$ such that if $q$ is in $Q_{0}$ and $q \subseteq R$, then for all $\alpha \neq \beta$ in the domain of $q$ and $\delta$ in $E$,

$$
\Delta_{A}(\alpha, \beta)<\delta \Leftrightarrow \Delta_{A}(q(\alpha), q(\beta))<\delta .
$$

Proof. Suppose that $\zeta$ is in $A$. Define $h(\zeta)$ to be the supremum of all countable ordinals of the form $\Delta_{A}\left(\beta, \beta^{\prime}\right)$ such that for some $\alpha$ and $\alpha^{\prime}$ in $A$ with $\alpha<_{A} \zeta<_{A} \alpha^{\prime}$, either both $(\alpha, \beta)$ and $\left(\alpha^{\prime}, \beta^{\prime}\right)$ or both $(\beta, \alpha)$ and $\left(\beta^{\prime}, \alpha^{\prime}\right)$ are in $R$. This supremum is countable by Fact 2.1 and the assumption that $A$ is Aronszajn. If $E$ is the club of those $\delta<\omega_{1}$ which are closed under $h$, then $E$ satisfies the conclusion of the lemma.

The following lemma will be needed in proving the countable chain condition of certain refinements of $Q_{0}$.

Lemma 3.4. (H) Suppose that $\left\langle q_{\xi}: \xi<\omega_{1}\right\rangle$ is a sequence of elements of $Q_{0}$ which all respect some fixed club $E \subseteq \omega_{1}$. Then there is an uncountable $\Gamma \subseteq \omega_{1}$ such that $q_{\xi} \cup q_{\eta}$ is in $Q_{0}$ whenever $\xi \neq \eta$ are in $\Gamma$.

Proof. It will be useful to first make a definition and adopt a notational convention which will be used later as well. If $\left\langle q_{\xi}: \xi<\omega_{1}\right\rangle$ is an uncountable sequence of disjoint elements of $Q_{0}$, it is possible by routine arguments (and the use of $(\mathrm{H})$ and Fact 2.4) to find an uncountable subset $\Gamma$ of $\omega_{1}$ such that:

(1) for $\xi$ in $\Gamma,\left|\operatorname{dom}\left(q_{\xi}\right)\right|$ does not depend on $\xi$;

\footnotetext{
${ }^{2}$ In this paper, $p \leq q$ means that $p$ is a stronger condition in a given forcing notion.
} 
(2) there is a $\gamma<\min (\Gamma)$ such that if $\xi$ is in $\Gamma, \alpha \neq \alpha^{\prime}$ are in $\operatorname{dom}\left(q_{\xi}\right)$, and $\Delta_{A}\left(\alpha, \alpha^{\prime}\right)<\xi$, then

$$
\begin{gathered}
\Delta_{A}\left(\alpha, \alpha^{\prime}\right)<\gamma \\
\Delta_{C}\left(q_{\xi}(\alpha), q_{\xi}\left(\alpha^{\prime}\right)\right)<\gamma ;
\end{gathered}
$$

(3) if $\xi<\eta$ are in $\Gamma, i<n, \alpha$ is the $i^{\text {th }}$ least element of $\operatorname{dom}\left(q_{\xi}\right)$ and $\alpha^{\prime}$ is the $i^{\text {th }}$ least element of $\operatorname{dom}\left(q_{\eta}\right)$, then

$$
\begin{gathered}
\gamma<\Delta_{A}\left(\alpha, \alpha^{\prime}\right)<\xi \\
\gamma<\Delta_{C}\left(q_{\xi}(\alpha), q_{\eta}\left(\alpha^{\prime}\right)\right)<\xi ;
\end{gathered}
$$

(4) if $\xi<\eta$ are in $\Gamma$, then every ordinal mentioned in $q_{\xi}$ is less than every ordinal mentioned in $q_{\eta}$.

Such a sequence is said to be in standard form (with uniform size $n$ ). If only one such sequence is under discussion and $\xi$ is in $\Gamma$, I will use $\xi_{i}$ and $q \xi_{i}$ to denote the $i^{\text {th }}$ $<_{A}$-least element of $\operatorname{dom}\left(q_{\xi}\right)$ and its respective image under $q_{\xi}$.

Returning to the proof, first refine to a sequence $\left\langle q_{\xi}: \xi \in \Gamma_{0}\right\rangle$ in standard form. Applying $(\mathrm{H}) n$ times, using the fact that $C^{2 n}$ is the union of countably many non decreasing relations, it is possible to find an uncountable $\Gamma \subseteq \Gamma_{0}$ such that if $\xi<\eta$ are in $\Gamma$, then for all $i<n, \xi_{i}<_{A} \eta_{i}$ iff $q \xi_{i}<_{C} q \eta_{i}$ iff $\xi_{0}<_{A} \eta_{0}$. It suffices to show that $\Gamma$ satisfies the conclusion of the lemma. To this end, let $\xi<\eta$ be in $\Gamma$ and let $i, j<n$.

First suppose that $\Delta\left(\xi_{i}, \xi_{j}\right) \geq \xi$. By definition of $\Gamma_{0}$, this implies $\Delta\left(\eta_{i}, \eta_{j}\right) \geq \eta$. Consequently, $\xi_{i}<_{A} \eta_{j}$ iff $\xi_{i}<_{A} \eta_{i}$ iff $q \xi_{i}<_{C} q \eta_{i}$ iff $q \xi_{i}<_{C} q \eta_{j}$ as desired. Notice that in the last equivalence we utilized that $q_{\eta}$ respects $\eta$.

Now suppose that $\Delta\left(\xi_{i}, \xi_{j}\right)<\xi$. Then $\Delta\left(\xi_{i}, \xi_{j}\right)=\Delta\left(\eta_{i}, \eta_{j}\right)$ and $\Delta\left(q \xi_{i}, q \xi_{j}\right)=$ $\Delta\left(q \eta_{i}, q \eta_{j}\right)$. Hence $\xi_{i}<_{A} \xi_{j}$ iff $\xi_{i}<_{A} \eta_{j}$ and $q \xi_{q}<_{C} q \xi_{j}$ iff $q \xi_{i}<_{A} q \eta_{j}$. This finishes the proof.

The lemma suggests that, for an appropriately chosen club $E \subseteq \omega_{1}$, we consider the collection $Q \subseteq Q_{0}$ of all $q$ which respect $E$. Such an $E$ will need to satisfy two conditions - one to ensure that $Q$ satisfies the c.c.c. and the other to guarantee that certain sets are dense in $Q$.

Definition 3.5. A club $E \subseteq \omega_{1}$ is fast enough for $A$ and $C$ if whenever $\left\langle q_{\xi}: \xi<\omega_{1}\right\rangle$ is a sequence of disjoint elements of $Q_{0}$ which respect $E$ and $\alpha<\omega_{1}$, there are $\xi \neq \eta$ greater than $\alpha$ such that $q_{\xi} \cup q_{\eta}$ respects $E$. An ordinal $\nu<\omega_{1}$ is said to be elementary for $A$ and $C$ iff there is a countable elementary submodel $N$ of $H\left(\omega_{2}\right)$ such that $A$ and $C$ are in $N$ and $\nu=N \cap \omega_{1}$.

If $E$ is fast enough for $A$ and $C$ and consists of elementary ordinals, then define $Q$ to be all elements of $Q_{0}$ which respect every element of $E$. It follows immediately from Lemma 3.4 and the definition of fast enough that $Q$ satisfies the countable chain condition.

We now turn to the task of finding a fast enough club for a pair $A$ and $C$.

Lemma 3.6. If $A$ is Countryman and $C$ is isomorphic to a suborder of $A$, then there is a club $E$ which is fast enough for $A$ and $C$. 
Proof. Since $A$ is Countryman, there are non decreasing relations $R_{n} \subseteq A^{2}$ which cover $A^{2}$. Applying Lemma 3.3 and intersecting clubs, it is possible to find a club $E$ such that if $q$ is an element of $Q_{0}$ which is a subset of some $R_{n}$, then for all $\alpha \neq \beta$ in the domain of $q$ and $\delta$ in $E$,

$$
\Delta_{A}(\alpha, \beta)<\delta \Leftrightarrow \Delta_{A}(q(\alpha), q(\beta))<\delta .
$$

It suffices to show that $E$ is fast enough for $A$ and $C$.

To this end, suppose that $\left\langle q_{\xi}: \xi<\omega_{1}\right\rangle$ is given sequence of elements of $Q_{0}$ which each respect $E$. Select an uncountable $\Gamma \subseteq \omega_{1}$, an $n<\omega$, and natural numbers $m_{i, j}$ for $i<j<n$ such that if $\xi$ is in $\Gamma$, then the domain of $q_{\xi}$ has cardinality $n$ and if $i, j<n$, then $\left(\xi_{i}, q \xi_{j}\right)$ is in $R_{m_{i, j}}$. It is now follows that if $\xi<\eta$ are in $\Gamma$, then $q_{\xi} \cup q_{\eta}$ respects $E$. In particular, $\Gamma$ satisfies the desired property with respect to $E$ and $\left\langle q_{\xi}: \xi<\omega_{1}\right\rangle$.

Hence if both $A$ and $C$ are Countryman, then $Q$ can be built in $V$ and an application of $\mathrm{MA}_{\aleph_{1}}$ to the dense sets provided by Lemma 3.10 below suffices to obtain the conclusion of Theorem 1.1. The next lemma indicates that fast enough clubs always exist in appropriate generic extensions by $\sigma$-closed forcing notions. The argument of $[1, \S 5]$ can be adapted to show that Baumgartner's forcing for adding a club by finite approximations also can be used to generate fast clubs.

Lemma 3.7. (H) Suppose $V[G]$ is a generic extension by a $\sigma$-closed forcing and $E \subseteq \omega_{1}$ is a club in $V[G]$ such that for every club $E^{\prime} \subseteq \omega_{1}$ in $V, E \backslash E^{\prime}$ is countable. Then $E$ is fast enough for every $A$ and $C$ in $V[G]$.

Remark 3.8. If the cardinality of $\mathscr{P}\left(\omega_{1}\right) \cap V$ is collapsed to $\aleph_{1}$ by a $\sigma$-closed forcing, then in the extension, there is a club satisfying the hypothesis of Lemma 3.7.

Proof. Suppose for a moment that $\left\langle q_{\xi}: \xi \in \Gamma\right\rangle$ is a sequence in standard form of disjoint elements of $Q_{0}$. Another such sequence $\left\langle q_{\xi}^{\prime}: \xi<\Gamma\right\rangle$ is $\Delta$-equivalent to $\left\langle q_{\xi}: \xi<\omega_{1}\right\rangle$ iff they have the same uniform size $n$ and for all $i, j<n$

$$
\begin{gathered}
\Delta\left(\xi_{i}, \eta_{j}\right)=\Delta\left(\xi_{i}^{\prime}, \eta_{j}^{\prime}\right) \\
\Delta\left(q \xi_{i}, q \eta_{j}\right)=\Delta\left(q \xi_{i}^{\prime}, q \eta_{j}^{\prime}\right) .
\end{gathered}
$$

(Here, e.g., $\xi_{i}^{\prime}$ and $q \xi_{i}^{\prime}$ have the obvious meaning.) The following claim follows easily from $[15,8.13]$.

Claim 3.9. If $G$ is generic for a $\sigma$-closed forcing, $A$ and $C$ are in $V$, and $\left\langle q_{\xi}: \xi \in \Gamma\right\rangle$ is an element of $V[G]$ which is an uncountable sequence of disjoint elements of $Q_{0}$, then there is an uncountable subsequence which is $\Delta$-equivalent to a sequence in $V$.

Finally notice that for a sequence $\left\langle q_{\xi}: \xi<\omega_{1}\right\rangle$ as above, there is a club $E_{\vec{q}}$ which is fast enough for the sequence. Hence a club $E$ as in the statement of the lemma is fast enough for all ground model sequences and therefore, by Claim 3.9, for all sequences in $V[G]$ as well.

Now we turn our attention to the relevant density lemma. The proof of this lemma is routine but somewhat tedious. Its proof was omitted in the analogous argument in $[21,2.1 .12]$. I have included it here for completeness and because this is where the 
(essential) assumptions of $\aleph_{1}$-density and non stationarity are used in the proof of Theorem 1.1.

Lemma 3.10. Suppose $A$ and $C$ are Aronszajn lines ( $C$ need not be Countryman). If $C$ is non stationary and $\aleph_{1}$-dense and $\alpha$ is in $A$, then the set of all $q$ in $Q$ such that $\alpha$ is in the domain of $q$ is dense in $Q$. Similarly, if $A$ is non stationary and $\aleph_{1}$-dense and $\beta$ is in $C$, then the set of all $q$ in $Q$ such that $\beta$ is in the range of $q$ is dense in $Q$.

Proof. By replacing the role of $A$ and $C$ and elements of $Q$ with their inverses, it suffices to prove the first half of the lemma. Assume that $C$ is non stationary and $\aleph_{1}$-dense and let $q$ be an element of $Q$ and $\alpha$ be in $A$. If $\xi<\omega_{1}$, let $\delta(\xi)$ be the greatest element of $E \cup\{0\}$ which is less than or equal to $\xi$. If $\alpha \neq \alpha^{\prime}$ are in $A$, then $\delta_{A}(\alpha, \beta)$ will be used to abbreviate $\delta\left(\Delta_{A}\left(\alpha, \alpha^{\prime}\right)\right)$. This is similarly defined for $\alpha \neq \alpha^{\prime}$ in $C$.

Let $\alpha_{0}$ and $\alpha_{1}$ be elements of $\operatorname{dom}(q) \cup\{-\infty,+\infty\}$ such that $\alpha_{0}<_{A} \alpha<_{A} \alpha_{1}$ in $A$ and there is no $\alpha^{\prime} \neq \alpha$ in $\operatorname{dom}(q)$ such that $\alpha_{0}<_{A} \alpha^{\prime}<_{A} \alpha_{1}$. We will focus on the case where neither $\alpha_{0}$ nor $\alpha_{1}$ are $\pm \infty$ since the argument applies with routine modification to the degenerate cases. There are now three cases depending on the relationship between $\delta_{A}\left(\alpha_{0}, \alpha\right)$ and $\delta_{A}\left(\alpha, \alpha_{1}\right)$.

If $\delta_{A}\left(\alpha_{0}, \alpha\right)=\delta_{A}\left(\alpha, \alpha_{1}\right)$, then observe that we also have

$$
\delta_{A}\left(\alpha_{0}, \alpha\right)=\delta_{A}\left(\alpha_{0}, \alpha_{1}\right)=\delta_{C}\left(q\left(\alpha_{0}\right), q\left(\alpha_{1}\right)\right) .
$$

Let $\nu$ denote this common value and let $\nu^{+}$be the least element of $E$ greater than $\nu$. Using elementarity of $\nu^{+}$and $\aleph_{1}$-density of $C$, select $\beta_{0}<_{C} \beta_{1}$ such that $\delta\left(\beta_{0}\right)=$ $\delta\left(\beta_{1}\right)=\nu$. Again using $\aleph_{1}$-density of $C$ and elementarity of $\nu^{+}$, choose an extension $\bar{q}$ of $q$ to $\alpha$ so that

$$
\begin{gathered}
\delta(\bar{q}(\alpha))=\delta(\alpha) \\
\beta_{0}<_{C} \bar{q}(\alpha)<_{C} \beta_{1} .
\end{gathered}
$$

Observe that $\delta_{C}\left(\bar{q}\left(\alpha_{i}\right), \bar{q}(\alpha)\right)$ is at least $\nu$ by Fact 2.1 and at most $\nu$ as witnessed by $\beta_{i}$. Transitivity of $<_{A}$ and $<_{C}$ implies $\bar{q}$ is in $Q_{0}$ and Fact 2.1 can be used to verify that $\bar{q}$ respects $E$ and hence is in $Q$.

Now suppose that $\delta_{A}\left(\alpha_{0}, \alpha\right) \neq \delta_{A}\left(\alpha, \alpha_{1}\right)$. As the argument is similar in both cases, I will only present the proof for the case $\delta_{A}\left(\alpha_{0}, \alpha\right)<\delta_{A}\left(\alpha, \alpha_{1}\right)$. By non stationarity of $C$ and arguing as in the previous case, there is a $\beta$ in $C$ such that $q\left(\alpha_{0}\right)<_{C} \beta<_{C} q\left(\alpha_{1}\right), \delta(\beta)=\delta(\alpha)$, and $\delta_{C}\left(\beta, q\left(\alpha_{1}\right)\right)=\delta_{A}\left(\alpha, \alpha_{1}\right)$. If $\bar{q}$ is an extension of $q$ to $\alpha$ so that $\bar{q}(\alpha)=\beta$, then $\bar{q}$ is in $Q$ as desired.

Finally, in Section 5 it will be useful to have the following lemma.

Lemma 3.11. $\left(\mathrm{MA}_{\aleph_{1}}\right)$ Suppose that $C$ is Countryman. Then there is an uncountable suborder $C_{0}$ of $C$ which is isomorphic to $C$ such that whenever $x$ is in $C \backslash C_{0}$, there is a set $H \subseteq C$ which is isomorphic to $\mathbb{Q}$ such that $x$ is in $H$ and no two elements of $H$ have an element of $C_{0}$ between them.

This can be accomplished by applying Theorem 1.3 to $A=C \times \mathbb{Q}$ for any $C$ (the above arguments show $\mathrm{MA}_{\aleph_{1}}$ is sufficient). This gives an embedding of $C \times \mathbb{Q}$ into $C$ and, setting $C_{0}$ equal to the image of $C \times\{0\}$, we have the desired suborder. 
Remark 3.12. The existence of a pair $C_{0} \subseteq C$ as in Lemma 3.11 does not require the assumption of $\mathrm{MA}_{\aleph_{1}}$. This can be achieved either by modifying the constructions of [21] or invoking Keisler's completeness theorem for $\mathscr{L}_{\omega_{1} \omega}(Q)[5]$.

\section{Families of Aronszajn subtrees and PFA}

In the proof that $\eta_{C}$ is universal, we will be interested in certain collections of subtrees of an Aronszajn tree T. In this section I will recall some the result of [6] which will be needed and also prove some additional related lemmas. For ease of reading, $T$ will denote an arbitrary Aronszajn tree for the duration of this section. A subset $U \subseteq T$ is a subtree if $U$ is uncountable and downward closed - whenever $s<t$ are in $T$ with $t$ in $U$, it follows that $s$ is in $U$.

Definition 4.1. A collection $\mathscr{F}$ of subtrees of $T$ is predense if whenever $U$ is a subtree of $T$, there is a $V$ in $\mathscr{F}$ such that $U \cap V$ is uncountable (and hence a subtree). If $\mathscr{F}$ is a collection of subtrees, then $\mathscr{F}^{\perp}$ will be used to denote the set of all subtrees $U$ of $T$ which have countable intersection with every element of $\mathscr{F}$.

Notice that trivially $\mathscr{F} \cup \mathscr{F}^{\perp}$ is predense. Also, it is trivial that $\left(\mathscr{F}^{\perp}\right)^{\perp}$ contains $\mathscr{F}$, though in general one does not expect equality to hold.

In order to motivate our interest in these definitions, suppose that $T$ is equipped with a lexicographic order and that $C$ is a Countryman line. Let $\pm \mathscr{F}$ consist of all subtrees $U$ of $T$ which are embeddable into $\pm C$ when equipped with the inherited lexicographic order. It should be clear that every element of $+\mathscr{F}$ has countable intersection with every element of $-\mathscr{F}$ or, equivalently, that $\pm \mathscr{F} \subseteq \mp \mathscr{F} \perp$. This follows from the basic property of Countryman lines mentioned in the introduction.

Recall the following theorem of [6].

Theorem 4.2. (PFA) If $\mathscr{F}$ is a collection of subtrees of $T$, then there are $\left\langle U_{\xi}: \xi<\right.$ $\left.\omega_{1}\right\rangle,\left\langle V_{\xi}: \xi<\omega_{1}\right\rangle$, and a club $E \subseteq \omega_{1}$ such that:

(1) For all $\xi<\omega_{1}$, either $U_{\xi}$ is in $\mathscr{F}$ or is empty and $V_{\xi}$ is in $\mathscr{F}^{\perp}$ or is empty.

(2) If $t$ is in $T$ with height $\delta$ in $E$, then either:

(a) there is a $\xi<\delta$ such that $t$ is in $V_{\xi}$ or

(b) there is a $\delta_{t}<\delta$ such that if $\nu$ is in $E$ with $\delta_{t}<\nu<\delta$, then there is a $\xi<\nu$ with $t \uparrow \nu$ in $U_{\xi}$.

If $\mathscr{F}=\left(\mathscr{F}^{\perp}\right)^{\perp}$, then it is possible to draw a stronger, simpler conclusion.

Theorem 4.3. (PFA) If $\mathscr{F}$ is a collection of subtrees such that $\mathscr{F}=\left(\mathscr{F}^{\perp}\right)^{\perp}$, then there is a sequence $\left\langle U_{\xi}: \xi<\omega_{1}\right\rangle$ of elements of $\mathscr{F} \cup \mathscr{F}^{\perp}$ and a club $E \subseteq \omega_{1}$ such that if $t$ is in $T$ of height $\delta$ in $E$, then there is $a \xi<\delta$ such that $t$ is in $U_{\xi}$.

Now we will return to our example.

Lemma 4.4. (PFA) Suppose that $+\mathscr{F}$ and $-\mathscr{F}$ are defined as above. If $U$ is a subtree of $T$ and $U$ has countable intersection with every element of $\pm \mathscr{F}$, then $U$ is in $\mp \mathscr{F}$ (i.e. $\pm \mathscr{F}^{\perp}=\mp \mathscr{F}$ ). 
Proof. Since $-C$ is also Countryman, it suffices to prove that $+\mathscr{F} \perp=-\mathscr{F}$. Suppose that $U$ is an arbitrary subtree of $T$. By Theorem 1.3, it suffices to prove that either $U$ has uncountable intersection with some element of $+\mathscr{F}$ or else every uncountable subset of $U$ contains an uncountable suborder which embeds into $-C$. Let $X$ be an arbitrary uncountable subset of $U$. By [10], there is a subset $Y$ of $X$ which is isomorphic to either $C$ or $-C$. If it is always the case that this set $Y$ is isomorphic to $-C$, then we are done.

If $Y$ is isomorphic to $C$, then I claim that the downward closure $V$ of $Y$ must be an element of $+\mathscr{F}$. Again, by appealing to Theorem 1.3, it is sufficient to show that if $Z$ is any uncountable subset of $V$, then $Z$ contains a subset embeddable into $C$. To see that this is indeed the case, let $Z \subseteq V$ be uncountable and select an uncountable $Z_{0} \subseteq Z$ which is an antichain in the tree order. For each $z$ in $Z_{0}$, let $\phi(z)$ be an element of $Y$ such that $z \leq \phi(z)$ in the tree order on $T$. It follows from the definition of lexicographic ordering that $\phi$ preserves the lexicographic order and hence embeds $Z_{0}$ into an isomorphic copy of $C$. This finishes the proof.

\section{PFA implies $\eta_{C}$ is universal}

I am now ready to argue that PFA implies that $\eta_{C}$ is universal. In this section $A$ and $C$ will be as in Section 2 and, for brevity, the subscript of $A$ in $T_{A}$ and $\Delta_{A}$ will be suppressed. Also, fix a $C_{0} \subseteq C$ as in Lemma 3.11.

Applying the results of Section 4, fix a sequence $\left\langle U_{\xi}: \xi<\omega_{1}\right\rangle$ of closed subtrees of $T$ and a club $E \subseteq \omega_{1}$ satisfying the conclusions of the Lemma 4.3. Let $\sigma_{\xi} \in\{+,-\}$ be such that $U_{\xi}$ is in $\sigma_{\xi} \mathscr{F}$ and pick $f_{\xi}: U_{\xi} \rightarrow \sigma_{\xi} C_{0}$ which is order preserving. Here we view the range of $f_{\xi}$ as a subset of $\zeta_{C}$. Define $\hat{U}_{\xi}$ for $\xi<\omega_{1}$ to be the set of all $s$ in $S$ with length in $E$ such that either $s$ is in $U_{\xi}$ or else the length of $s$ is at most the least element of $E$ greater than $\Delta\left(s, U_{\xi}\right)$. Notice that, for a fixed $\xi<\omega_{1}$, every interval of $\hat{U}_{\xi}$ which is disjoint from $U_{\xi}$ is countable. It follows that $f_{\xi}$ can be extended to an order preserving map from $\hat{U}_{\xi}$ to $C$. This extension will also be denoted by $f_{\xi}$.

Since $A$ is isomorphic to a suborder of $T$, it is sufficient to define an order preserving map from $T$ into $\eta_{C}$. If $t$ is in $T$, define two finite sequences $\left\langle\xi_{i}: i<k\right\rangle$ and $\left\langle\nu_{i}: i<k\right\rangle$ of ordinals as follows. Set $\nu_{0}$ equal to the least element of $E$. If $\nu_{i}$ has been defined, $\xi_{i}$ is the least ordinal such that $t\left\lceil\nu_{i}\right.$ is in $U_{\xi_{i}}$. If $\xi_{i}$ has been defined and $t$ is not in $U_{\xi_{i}}$, let $\nu_{i+1}$ be the least element of $E$ such that $t\left\lceil\nu_{i+1}\right.$ is not in $U_{\xi_{i}}$. Let $k$ be minimal such that $t$ is in $U_{\xi_{k-1}}$. Notice that, for all $i<k, t\left\lceil\nu_{i}\right.$ is in $\hat{U}_{\xi_{i}}$. Define $\Phi(t)$ in $\eta_{C}$ as follows. If $i<k$, put $\Phi(t)(i)=f_{\xi_{i}}\left(t\left\lceil\nu_{i}\right)\right.$ and if $i \geq k$, put $\Phi(t)(i)=0$.

It now suffices to show that $\Phi$ preserves order. Suppose that $s<\operatorname{lex} t$ are in $T$ and let $\gamma$ be the least ordinal such that $s(\gamma) \neq t(\gamma)$. The sequences of $\xi_{i}$ 's and $\nu_{i}$ 's which are constructed for $s$ and $t$ coincide at least until $\nu_{i}$ is greater than $\gamma$; let $i$ be maximal such that $\nu_{i} \leq \gamma$. We know that $s \uparrow \gamma=t \uparrow \gamma$ is in $U_{\xi}$ and therefore that $s \uparrow(\gamma+1)$ and $t \uparrow(\gamma+1)$ are in $\hat{U}_{\xi}$.

Observe that since $s\left\lceil\nu_{i}=t\left\lceil\nu_{i}, \Phi(s)(j)=\Phi(t)(j)\right.\right.$ for all $j<i$. Also, $s<_{\operatorname{lex}} t$ iff $s\left\lceil\nu_{i+1}^{s}<_{\operatorname{lex}} t\left\lceil\nu_{i+1}^{t}\right.\right.$ iff $s \uparrow(\gamma+1)<_{\operatorname{lex}} t \uparrow(\gamma+1)$. It follows that $s<_{A} t$ iff $\Phi(s)(i)<_{\zeta_{C}} \Phi(t)(i)$ iff $\Phi(s)<_{\eta_{C}} \Phi(t)$. Since $s$ and $t$ were arbitrary, $\Phi$ preserves order. 


\section{Concluding remarks and questions}

As mentioned in the introduction, the results of this paper suggest an analogy between the relationship of $\omega$ and $-\omega$ to $\eta=\operatorname{otp}(\mathbb{Q})$ and the relationship of $C$ and $-C$ to $\eta_{C}$ under the assumption of PFA. In this analogy, $\zeta_{C}$ corresponds to the order type $\zeta$ of $\mathbb{Z}$. Notice that, just as any linear order which does not contain $\eta$ must contain a non empty interval which is embeddable into $\omega$ or $-\omega$, we have the following proposition.

Proposition 6.1. (PFA) If $A$ is an Aronszajn line, then either $A$ is bi-embeddable with $C$ or else $A$ contains a non empty interval which is bi-embeddable with $C$ or $-C$.

If we define an Aronszajn line to be fragmented ${ }^{3}$ if it does not contain a copy of $\eta_{C}$, then the above analogy extends to give a strong connection between the (countable) scattered linear orders and the fragmented Aronszajn lines. In particular, one can associate a rank to a fragmented Aronszajn line which corresponds to how many applications of a derivative operation are necessary in order to trivialize it.

Laver has shown the countable linear orders are well quasi-ordered by embeddability [9] and the above analogy suggests the following conjecture.

Conjecture 6.2. (PFA) The Aronszajn lines are well quasi-ordered by embeddability.

This could be verified using the methods of [9] if one could prove the following from PFA (see [9] for undefined notions): If $Q$ is a better quasi-order and $C$ is a Countryman line, then $Q^{C}$ is a better quasi-order.

Finally, it should be noted that some large cardinal assumptions are likely necessary in order to establish the relative consistency of the results in this paper. The results of [6] show that all of the conclusions mentioned in this paper can be established consistent relative to the existence of a Mahlo cardinal. The conclusion of Theorem 4.2 implies that there are no Kurepa trees and hence has the consistency strength of at least an inaccessible cardinal. It is an open problem, however, whether the conclusion of Theorem 1.2 has any non-trivial consistency strength. It is also an intriguing problem whether the conclusion of Theorem 1.2 implies $|\mathbb{R}| \leq \aleph_{2}$.

\section{Acknowledgments}

This research was supported by NSF grants DMS-0401893 and DMS-0757507. I would like to thank Max Burke for his careful reading and review of [10]. The presentation in Section 3 owes much to my conversations with him while he was preparing the review.

\section{References}

[1] U. Abraham and S. Shelah, Isomorphism types of Aronszajn trees, Israel J. Math. 50 (1985), no. $1-2,75-113$

[2] J. Baumgartner, Topological properties of Specker types, Notices Amer. Math. Soc. 22 (1975) 219.

[3] M. Goldstern and S. Shelah, The Bounded Proper Forcing Axiom, J. Symbolic Logic 60 (1995), no. $1,58-73$.

[4] T. Jech, Set theory, Perspectives in Mathematical Logic, Springer-Verlag, Berlin, second edition (1997).

\footnotetext{
${ }^{3}$ This definition is only appropriate under a hypothesis such as PFA.
} 
[5] H. J. Keisler, Logic with the quantifier "there exist uncountably many", Ann. Math. Logic 1 (1970) 1-93.

[6] B. König, P. Larson, J. T. Moore, and B. Veličković, Bounding the consistency strength of a five element linear basis, Israel J. Math. 164 (2008), 1-18.

[7] K. Kunen, An introduction to independence proofs, Vol. 102 of Studies in Logic and the Foundations of Mathematics, North-Holland (1983).

[8] D. Kurepa, Ensembles ordonnés et ramifiés, Publ. Math. Univ. Belgrade 4 (1935) 1-138.

[9] R. Laver, On Fraïssé's order type conjecture, Ann. of Math. (2) 93 (1971) 89-111.

[10] J. T. Moore, A five element basis for the uncountable linear orders, Annals of Mathematics (2) 163 (2006), no. 2, 669-688.

[11] - $\omega_{1}$ and $\omega_{1}$ may be the only minimal uncountable order types, Michigan Math. Journal 55 (2007), no. 2, 437-457.

[12] S. Shelah, Decomposing uncountable squares to countably many chains, J. Combinatorial Theory Ser. A 21 (1976), no. 1, 110-114.

[13] — Proper and improper forcing, Springer-Verlag, Berlin, second edition (1998), ISBN 3-540-51700-6.

[14] M. Souslin, Problème 3, Fund. Math. 1 (1920) 223

[15] S. Todorcevic, Trees and linearly ordered sets, in Handbook of set-theoretic topology, 235-293, North-Holland, Amsterdam (1984).

[16] - Partitioning Pairs Of Countable Ordinals, Acta Math. 159 (1987), no. 3-4, 261-294.

[17] - Partition Problems In Topology, Amer. Math. Soc. (1989).

[18] — Aronszajn orderings, Publ. Inst. Math. (Beograd) (N.S.) 57(71) (1995) 29-46. Đuro Kurepa memorial volume.

[19] — A classification of transitive relations on $\omega_{1}$, Proc. London Math. Soc. (3) 73 (1996), no. $3,501-533$.

[20] — Basis problems in combinatorial set theory, Doc. Math. Extra Vol. II (1998) 43-52.

[21] - Walks on ordinals and their characteristics, Vol. 263 of Progress in Mathematics, Birkhäuser (2007).

[22] W. H. Woodin, The Axiom of Determinacy, Forcing Axioms, and the Nonstationary Ideal, Logic and its Applications, de Gruyter (1999).

Department of Mathematics, Cornell University, Ithaca, NY 14853-4201, USA

E-mail address: justin@math.cornell.edu 